circadian reprogramming, and what are the mediators of CR-induced circadian reprogramming? The observed enrichment of circadian genes related to $\mathrm{NAD}^{+}$metabolism, protein acetylation, and stress resistance points to the sirtuin family of $\mathrm{NAD}^{+}$-dependent deacetylases as potential mediators of reprogramming. Sirtuins are known to extend mammalian lifespan and healthspan ${ }^{15,16}$, mediate aspects of the $\mathrm{CR}$ response $\mathrm{e}^{3,4}$, and have increasingly been appreciated as stress-resistance regulators ${ }^{4,7,9,17}$. Fittingly, several sirtuins have been implicated in circadian control ${ }^{18}$ and there is some degree of overlap between SIRT1-dependent hepatic circadian genes and aging- or CR-associated hepatic circadian genes ${ }^{5}$. Further studies are needed to determine whether overexpression of SIRT1 or other mammalian sirtuins (SIRT2-SIRT7) prevents aging-associated circadian reprogramming and whether CR induces circadian reprogramming in the absence of sirtuins.

It is now widely accepted that the general cause of aging is the accumulation of cellular damage ${ }^{19,20}$. Aging-associated stress resistance is particularly relevant to adult stem cells, which persist throughout the organismal lifespan to repair and maintain tissues ${ }^{7,9}$. The aging-associated circadian reprogramming of both homeostasis and stressresistance genes observed in adult stem cells suggests that increased cellular damage is a driver of aging-associated circadian reprogramming. Given that aging-associated accumulation of DNA damage in stem cells originates from exposure to mitochondrial stress ${ }^{6}$ and that the mitochondrial protective programs are repressed in aged adult stem cells $^{7,9}$, it is tempting to speculate that reactivating the mitochondrial protective programs may provide a means to reduce the accumulation of cellular damage and reverse aging-associated circadian reprogramming.

\section{ACKNOWLEDGMENTS}

D.C. is supported by NIH grant R01DK101885, the National Institute of Food and Agriculture, the PackerWentz Endowment and the Chau Hoi Shuen Foundation. R.O. is supported by the ITO Scholarship and the Honjo International Scholarship.

\section{COMPETING FINANCIAL INTERESTS}

The authors declare no competing financial interests.
1. Luo, H., Chiang, H.H., Louw, M., Susanto, A. \& Chen, D. Trends Endocrinol. Metab. 28, 449-460 (2017).

2. Weindruch, R. \& Walford, R.L. The Retardation of Aging and Disease by Dietary Restriction (Charles C. Thomas Publisher, 1988).

3. Chen, D., Steele, A.D., Lindquist, S. \& Guarente, L. Science 310, 1641 (2005).

4. Qiu, X., Brown, K., Hirschey, M.D., Verdin, E. \& Chen, D. Cell Metab. 12, 662-667 (2010)

5. Sato, S. et al. Cell 170, 664-677 (2017).

6. Solanas, G. et al. Cell 170, 678-692 (2017)

7. Brown, K. et al. Cell Rep. 3, 319-327 (2013).

8. Goodell, M.A. \& Rando, T.A. Science 350, 1199-1204 (2015).

9. Mohrin, M. et al. Science 347, 1374-1377 (2015).

10. Kondratov, R.V., Kondratova, A.A., Gorbacheva, V.Y., Vykhovanets, O.V. \& Antoch, M.P. Genes Dev. 20, 1868-1873 (2006).

11. Nakamura, T.J. et al. J. Neurosci. 31, 10201-10205 (2011).

12. Sellix, M.T. et al. J. Neurosci. 32, 16193-16202 (2012).

13. Geyfman, M. et al. Proc. Natl. Acad. Sci. USA 109, 11758-11763 (2012).

14. Stringari, C. et al. Cell Rep. 10, 1-7 (2015).

15. Finkel, T., Deng, C.X. \& Mostoslavsky, R. Nature 460 587-591 (2009)

16. Kanfi, Y. et al. Nature 483, 218-221 (2012).

17. Shin, J. et al. Cell Rep. 5, 654-665 (2013).

18. Masri, S. \& Sassone-Corsi, P. Sci. Signal. 7, re6 (2014).

19. López-Otín, C., Blasco, M.A., Partridge, L., Serrano, M. \& Kroemer, G. Cell 153, 1194-1217 (2013).

20. Vijg, J. \& Campisi, J. Nature 454, 1065-1071 (2008).

\title{
A tripartite interface that regulates vesicle fusion
}

In neurons, SNARE proteins mediate the fusion of neurotransmittercontaining vesicles with the plasma membrane, leading to the release of neurotransmitters upon action potential. To prime fusion, SNARE proteins in the vesicle membrane (synaptobrevin-2) and in the target membrane (SNAP-25 and syntaxin-1A) form a complex characterized by a core fourhelix bundle, called the SNARE complex (pictured; synaptobrevin-2 is dark blue, SNAP-25 is green and syntaxin-1A is red).

However, SNARE proteins do not work alone. In fact, vesicle fusion is stringently regulated, both to prevent spontaneous release and to ensure that neurotransmitter release occurs in a fast and synchronous manner upon stimuli. $\mathrm{Ca}^{2+}$ influx, induced by action potential, triggers the fusion of vesicles docked to the cytoplasmic membrane. The $\mathrm{Ca}^{2+}$ sensor in this process is synaptotagmin-1 (Syt1), which has two $\mathrm{Ca}^{2+}$-binding domains, $\mathrm{C} 2 \mathrm{~A}$ and $\mathrm{C} 2 \mathrm{~B}$. Another regulator is complexin (Cpx), which is required to suppress spontaneous fusion.

Both Syt1 and Cpx interact with the SNARE complex and previous structural work has revealed details of those binary interactions, but the interplay between the two factors remained unclear. Now, Zhou et al. (Nature http://dx.doi.org/10.1038/nature23484) have determined the structure of the prefusion complex containing SNAREs, Syt1 and Cpx. Along with extensive analysis in neuronal cells, the work uncovers previously unknown interactions and provides major insights into the complex regulation of neuronal vesicle fusion.

Unexpectedly, the new structural data reveal two Syt1 molecules interacting with the SNARE complex via different interfaces, with their C2B domains bound on opposite sides of the four-helix bundle. The first C2B domain (gray) binds the SNARE complex via the previously known, or primary, interface. The second C2B domain (orange) interacts with the SNARE complex and with Cpx (light blue), in what the authors call a 'tripartite interface'. This interface (marked by a dashed box in the figure) involves the conserved HA $\alpha$-helix in $\mathrm{C} 2 \mathrm{~B}$, which extends the Cpx $\alpha$-helix that interacts with the SNARE complex.

Mutagenesis analyses of the tripartite interface show that it is required for $\mathrm{Ca}^{2+}$-triggered synchronous release by neurons, as is the primary interface. Thus, both binding interfaces are essential to reach the primed prefusion state captured in the new structure. However, the tripartite interface also serves to 'lock' the complex in a primed state; thus, Syt1 and Cpx cooperate to prevent calciumindependent vesicle fusion. The tripartite interface would be unlocked upon $\mathrm{Ca}^{2+}$ binding to the $\mathrm{C} 2$ domains, which would likely result in release of the Syt $1 \mathrm{C} 2 \mathrm{~B}$ domain involved in the tripartite complex and conformational rearrangements of the remaining complex. This proposed unlocking mechanism remains to be confirmed, but the present findings can already explain the phenotypes of Cpx or Syt1 knockouts and of dominant-negative Syt1 mutations. 